\title{
A Novel Approach to Control of Multilevel Converter Using Wavelets Transform
}

\author{
A. Jan Iwaszkiewicz ${ }^{1}$, B. Jacek Perz ${ }^{1}$ \\ ${ }^{1}$ The Electrotechnical Institute, Gdansk Branch, Poland \\ 80-557 Gdansk, Narwicka 1, Poland \\ phone:+48 58 3431291, fax+48 58 3431295, e-mail: A.jan.iwaszkiewicz@iel.gda.pl, B.jacek.perz@iel.gda.pl
}

\begin{abstract}
The paper presents an approach to the useful theory concerning the problem of generating high quality AC voltage and current waveforms in multilevel converters. The objective is to describe a novel proposal of $\mathrm{AC}$ waveforms synthesis based on applying analytical methods using sets of orthogonal wavelets. The discussion includes a short survey of Haar wavelet and a proposal of output waveforms synthesis based on wavelet transform. The comparison of the proposed method with standard solution is discussed. It has been done by use of such criteria as: average square error and total harmonic distortion (THD).
\end{abstract}

\section{Key words}

Multilevel converter, Haar wavelet, wavelet transform, wavelet waveforms synthesis.

\section{Introduction}

In contemporary industry and public area a great demand has been appeared for higher power devices converting electric energy. The devices like voltage and current converters which are able to control and to supply diverse equipment working in power range of hundreds $\mathrm{kW}$ and more. These devices have to fulfil definite and diversified requirements what implies diversified purposes and methods of electric energy conversion. For instance in $\mathrm{AC}$ drives where the possibility of speed regulation is essential, the converter e.g. a voltage or current source inverter (VSI or CSI) has to assure the adjustment of output waveform fundamental level and frequency $[1,2]$.

There are many other industrial applications e.g. uninterruptible power suppliers (UPS) or distributed power generation systems, where the essential demand is to generate 50 or $60 \mathrm{~Hz}$ sinusoidal voltage waveforms. The quality of generated waveforms, especially the Total Harmonic Distortion factor (THD), should comply with appropriate standards. Even if there is a need of output waveform adjustment, the output parameters have to be adjusted in a relatively small range.

In many devices like UPSs, active filters or voltage regulators in electric energy grids the main and most important features are: high quality of output waveforms, output stability and efficiency of the device $[4,5,6]$. Similar requirements are to be fulfilled in converters applied in renewable energy systems. In wind farms the conversion of produced energy is inevitable in order to solve the problem of unbalanced voltage levels and frequency synchronization of many separated sources. The similar problem appears in static DC sources like photovoltaic farms and fuel cells. The resulting DC power should be converted to AC form using power electronics converters.

During the first period of power electronics evolution a two-level inverter was considered as the most suitable device used to energy conversion in aforesaid applications. The features and performance as well as drawbacks and limitations of the two-level inverter have been largely recognized and verified in practice. Latest achievements in power semiconductor technology permit to work with higher switching frequency, but fast switching accompanying the PWM control causes power losses in switching elements thus cutting inverter efficiency down [7].

The range of the output power is a very important and evident limitation of two-level inverters. The total power is determined by the semiconductor switches properties. This essential disadvantage can be reduced using multilevel inverters. Recently, the multilevel inverters have emerged as a new and very important class of converters. Thanks to their promising performance, multilevel inverters are becoming more and more an alternative to conventional two-level inverters. They permit to overcome the problem of limited power and to shape output waveforms. A result is that many multilevel converters have been applied in industry.

The development of multilevel converters comprises an area of research for new topologies, control strategies and theory. Producing the required voltage or current waveforms is possible in many ways: e.g. sinusoidal PWM, selective harmonic elimination, space-vector modulation (SVM) or shaping the stepped voltage or current $[8,9,10]$. Important works and studies concern the subject of frequency adjustment. Diverse methods of converters' control such as computing the adequate switching angles of stepped waveforms or cancelling specified harmonics have been developed [11]. In all solutions a large number of semiconductor switches is needed. The switches are not charged equally and their admissible charge parameters are not fully utilized. It results in decreasing of converter efficiency which is a serious disadvantage especially in higher power applications.

The aforementioned disadvantages can be slightly reduced by use of novel converters topologies as well as mathematical tools aided control strategies. The paper deals with technique of shaping the stepped output waveforms in multilevel converters. A mathematical approach to the control strategy based on wavelets transform is presented. The output waveform synthesis is accomplished using set of orthogonal wavelets. The discussion includes such mathematical tools like Haar wavelet trans- 
form. The comparison of methods and their impact on topology is discussed in chapter 4 . The comparative analysis has been done by use of three criteria: number of DC sources needed, average square error and THD factor.

\section{Haar wavelet function}

The wavelets is a term for mathematical functions, which allow the analysis of signals in different time scale and with different resolution. The term ,wavelets" is the direct translation of French term ,ondelettes”, which means ,little waves”. Thanks to the adjustable ,scope of the view" the wavelets can be used to distinguish and analyse small and big details of the investigated process. Especially they are useful in analyse of discontinuous or stepped processes. The wavelets have been applied in many not directly related areas like seismology, video analysis, quantum mechanics or electronics $[12,13,14$, $15,16,17]$.

Till now the wavelets have been used mainly for analysis of processes or signals based on decomposition of the elements of the processes. The following considerations will prove that wavelets can be also useful in composition of the power electronics signals and structures.

For this purpose the Haar wavelets have been adapted. The fundamental Haar wavelet can be constructed by transforming the following scaling function $\varphi(t)$ :

$$
\varphi(t)=\left\{\begin{array}{ll}
1 & \text { for } 0 \leq t<1, \\
0 & \text { for other } t
\end{array}\right\}
$$
tions:

The composition of two consecutive scaling func-

$$
\varphi(2 t)=\left\{\begin{array}{ll}
1 & \text { for } 0 \leq t<0,5), \\
0 & \text { for other } t
\end{array}\right\}
$$

and

$$
\varphi(2 t-1)=\left\{\begin{array}{ccc}
1 & \text { for } & 0,5 \leq t<1), \\
0 & \text { for } & \text { other } t
\end{array}\right\}
$$

creates the fundamental Haar wavelet: $\psi(t)=\varphi(2 t)-\varphi(2 t-1)$ which can be defined as follows:

$$
\psi(t)=\left\{\begin{array}{c}
1 \text { for } 0 \leq t<0.5 \\
-1 \text { for } \frac{1}{2} \leq t<1 \\
0 \text { for other } t
\end{array}\right\}
$$

The scaling functions and the fundamental Haar wavelet are presented in Fig. 1.

Introducing two parameters: $m$ - scale factor and $n-$ displacement factor it is possible to write the family of wavelets:

$\psi_{m n}(t)=\frac{1}{\sqrt{2^{m}}} \psi\left(2^{-m} t-n\right)$ for $m, n=\ldots,-2,-1,0,1,2, \ldots$
The equation defines an orthonormal wavelet family with dyadic scaling.
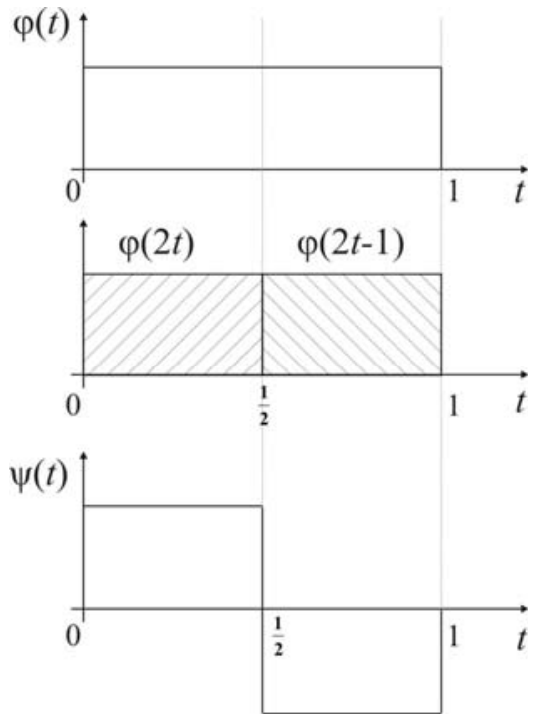

Fig. 1. The scaling functions and and the fundamental Haar wavelet.

For given $m$ the wavelet scale is $2 m$. The scale factor $m$ - settles the width and amplitude of the wavelet, and the displacement factor $n$ - settles the wavelet position on the time axis.

The fundamental Haar wavelet corresponds to the factors: $m=0$ and $n=0$ and can be denoted as $\psi(t)=\psi_{00}(t)$.

\section{Wavelet waveforms synthesis}

The Haar wavelet form is similar to the form of the voltage or current pulse that can be obtained using simple one-phase inverter i.e. H-bridge cell. The displacement and width of the wavelet can be freely controlled. Thanks to these properties it is possible to apply wavelets in power electronics e.g. to form the output stepped waveforms of multilevel converters.

Let us define the scaling function $\varphi(x)$ in an interval $x \in<0,2 \pi)$ :

$$
\varphi(x)=\left\{\begin{array}{ccc}
1 & \text { for } & 0 \leq x<2 \pi \\
0 & \text { for other } x
\end{array}\right\}
$$

The fundamental proposed wavelet is defined as the Haar one alike:

$$
\psi(x)=\left\{\begin{array}{l}
1 \text { for } 0 \leq x<\pi \\
-1 \text { for } \pi \leq x<2 \pi \\
0 \text { for other } x
\end{array}\right\}
$$

The wavelet determines one period of the rectangular wave and is mother function introducing a family of wavelets:

$$
\psi_{m n}(x)=\psi\left(2^{-m} x-2 \pi n\right) \text { for } m, n=\ldots,-2,-1,0,1,2, \ldots
$$

The wavelet scale is done as $2^{m} 2 \pi$ and its displacement on the $\mathrm{x}$ axis is determined as $n$-times $2^{m+1} \pi$. The $m$ factor scales not only the wavelet but the amplitude too. 
The scaling function and a few wavelets have been presented in Fig. 2.

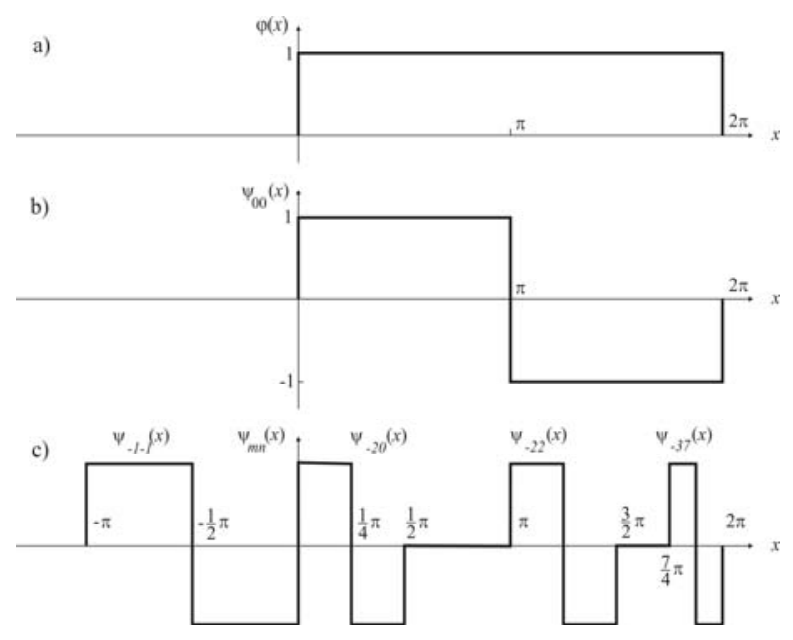

Fig. 2. The scaling function $\varphi(x)$ and wavelets $\psi_{m n}(x)$ :

a) scaling function $\varphi(x)$, b) fundamental wavelet $\psi_{00}(x)$,

c) wavelets $\psi_{-1-1}(x), \psi_{-20}(x), \psi_{-22}(x), \psi_{-37}(x)$.

All wavelets $\psi_{m n}(x)$ are orthogonal in the interval $x \in<0,2 \pi)$. The defined statement creates a family of orthogonal functions and can determine a basis of wavelet transform. A continuous wavelet transform is defined as:

$$
W f(m, n)=\int_{-\infty}^{\infty} f(x) \psi_{m n}(x) \mathrm{d} x
$$

and presents itself a scalar product of a function $f(x)$ and function $\psi_{m n}(x)$. A complete reconstruction of the function $f(x)$ takes place when the inverse wavelet transform is applied

$$
f(x)=C \int_{-\infty}^{\infty} \int_{-\infty}^{\infty} W f(m, n) \psi_{m n}(x) \mathrm{d} m \mathrm{~d} n
$$

The symbol $\mathrm{C}$ denotes a constant which can be calculated from Fourier transform of the function $\psi_{m n}(x)$.

For applications more comfortable is to use a discrete inverse wavelet transform which is defined by the following equation:

$$
f(x)=C \sum_{m=-\infty}^{\infty} \sum_{n=-\infty}^{\infty} W f(m, n) \psi_{m n}(x)
$$

It could be written as a sum of wavelets $\psi_{m n}(x)$ multiplied by coefficients $a_{m n}$

$$
f(x)=\sum_{m=-\infty}^{\infty} \sum_{n=-\infty}^{\infty} a_{m n} \psi_{m n}(x)
$$

The coefficients $a_{m n}$, called wavelet coefficients, are scalar products of the function $f(x)$ and wavelets $\psi_{m n}(x)$. In the interval $x \in<0,2 \pi$ ) they are given as

$$
a_{m n}=C_{m} \int_{0}^{2 \pi} f(x) \psi_{m n}(x) \mathrm{d} x
$$

The constant $\mathrm{C}_{m}$ is only dependent on coefficient $m$ and has the same value for different $n$.
Denoting

$$
f_{m n}(x)=a_{m n} \psi_{m n}(x)
$$

it is possible to write $f_{\Psi}(x)$ as a sum of components $f_{m n}(x)$ :

$$
f_{\psi}(x)=\sum_{m=-3}^{m=0} \sum_{n=0}^{2^{-m}-1} f_{m n}(x)
$$

The components $f_{m n}(x)$ present component wavelets, amplitude and phase of which is determined by coefficients $a_{m n}$.

Practically in power electronics applications, the approximation of a sine wave should be realized using a finite number of wavelets. Natural aspiration of designers is to utilize the possibly lowest number of components. The accuracy of approximation depends on it. In mathematics the accuracy is determined as an average square error $\delta$, a very useful criterion destined to that purpose. In power electronics the most important criterion of the approximated waveforms is THD factor.

Let us denote by $f_{\Psi}(x)$ a waveform approximating the function $f(x)=\sin (x)$ in the interval $x \in<0,2 \pi)$. Assuming that $f_{\Psi}(x)$ forms a combination of wavelets determined by index $m=-3,-2,-1,0$ it can be written as a sum:

$$
f_{\psi}(x)=\sum_{m=-3}^{m=0} \sum_{n=0}^{2^{-m}-1} a_{m n} \psi_{m n}(x)=\sum_{m=-3}^{m=0} \sum_{n=0}^{2^{-m}-1} f_{m n}(x)
$$

The coefficients $a_{m n}$ have been collected in Table 1 .

TABLE 1: The wavelet coefficients $a_{m n}$.

\begin{tabular}{|c||c|c|c|c|c|c|c|c||}
\hline \hline$a_{m n}$ & $n=0$ & $n=1$ & $n=2$ & $n=3$ & $n=4$ & $n=5$ & $n=6$ & $n=7$ \\
\hline \hline$a_{0 n}$ & 0,6366 & - & - & - & - & - & - & - \\
\hline$a_{-1 n}$ & 0 & 0 & - & - & - & - & - & - \\
\hline$a_{-2 n}$ & $-0,2637$ & 0,2637 & 0,2637 & $-0,2637$ & - & - & - & - \\
\hline$a_{-3 n}$ & $-0,1791$ & $-0,0742$ & 0,0742 & 0,1791 & 0,1791 & 0,0742 & $-0,0742$ & $-0,1791$ \\
\hline \hline
\end{tabular}

Successive steps of wavelet approximation $f_{\Psi k}(x)$ for $k=1,2,3$ have been presented in Fig. 3 - 5 .

First step of reconstruction creates function $f_{\Psi 1}(x)$ as a set of wavelets

$$
f_{\psi_{1}}(x)=\sum_{m=-2}^{m=0} \sum_{n=0}^{2^{-m}-1} f_{m n}(x)=f_{-20}+f_{-21}+f_{-22}+f_{-23}+f_{-10}+f_{-11}+f_{00}
$$

in which two component wavelets are equal to zero according to the Table 1. The resulting waveforms are presented in Fig. 3.

Fig. 4 presents the result of the second step of approximation in which a few (but not all) wavelets $f_{-3 n}$ have been added to the function $f_{\Psi 1}(x)$. Function $f_{\Psi_{2}}(x)$ creates a composition of following wavelets

$$
f_{\psi_{2}}(x)=f_{00}+f_{-20}+f_{-21}+f_{-22}+f_{-23}+f_{-30}+f_{-33}+f_{-34}+f_{-37}
$$


Finally taking all wavelets for scale coefficients: $m=0, m=-2, m=-3$, the approximating function presents itself as a set of wavelets:

$$
f_{\psi_{3}}(x)=f_{00}+f_{-20}+f_{-21}+\ldots+f_{-23}+f_{-30}+f_{-31}+\ldots+f_{-36}+f_{-37}
$$

The resulting waveform is presented in Fig. 5.

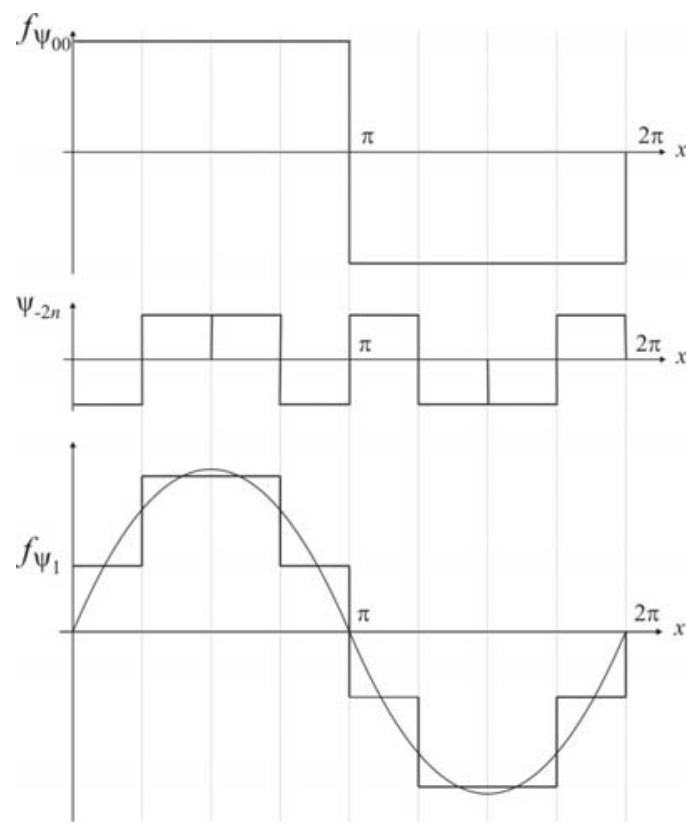

Fig. 3. First step of wavelet approximation: $f_{\psi_{1}}(x)=f_{-20}+f_{-21}+f_{-22}+f_{-23}+f_{00}$.
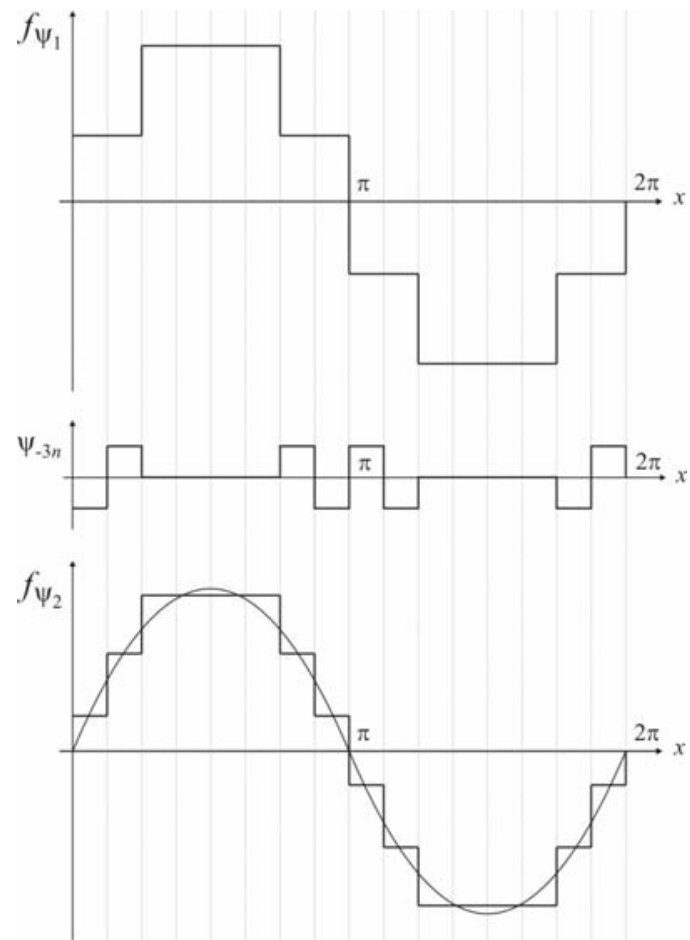

Fig. 4. Second step of wavelet approximation: $f_{\psi_{2}}(x)=f_{\psi 1}+f_{-30}+f_{-33}+f_{-34}+f_{-37}$.
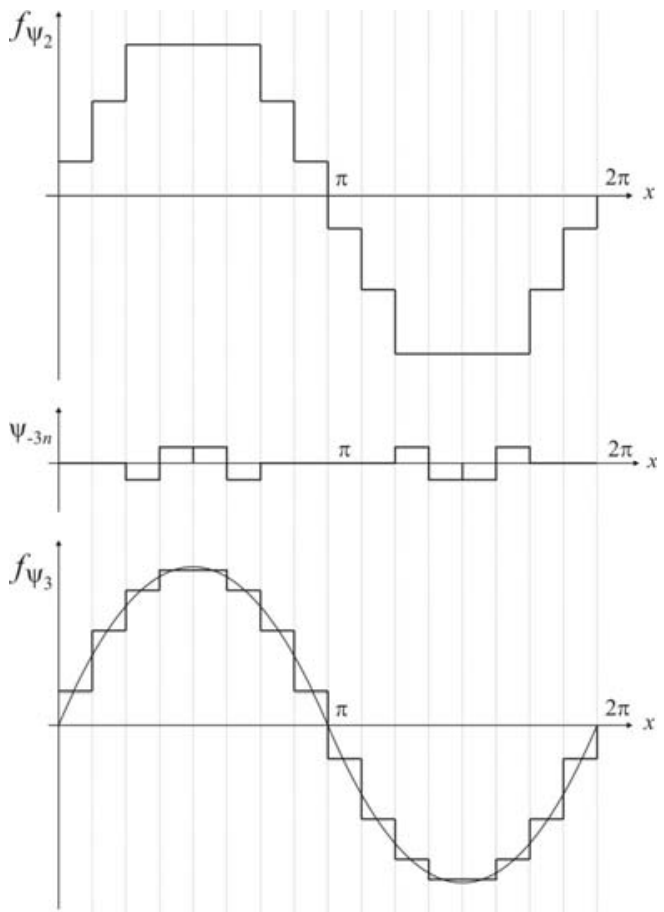

Fig. 5. Third step of wavelet approximation: $f_{\psi_{3}}(x)=f_{\psi^{2}}+f_{-31}+f_{-32}+f_{-35}+f_{-36}$.

Three approximating functions $f_{\Psi_{1}}(x), f_{\Psi_{2}}(x)$, $f_{\psi_{3}}(x)$ and related spectra have been presented below in Fig. 6 - 11. The adequate values of THD factors and average square errors $\delta$ have been also included.

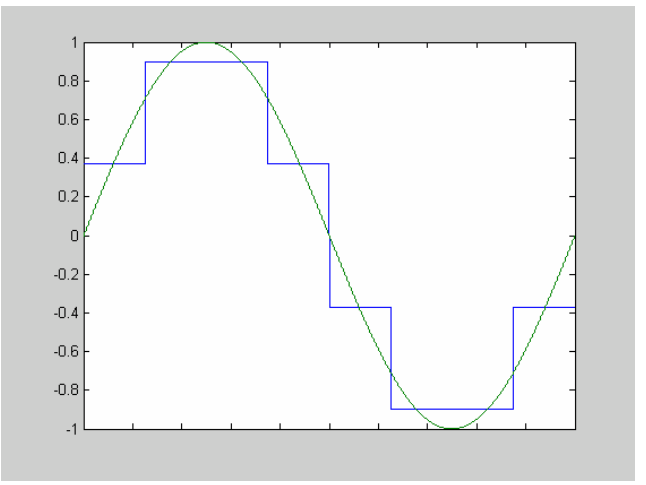

Fig. 6. Waveform of function $f_{\Psi_{1}}(x)$

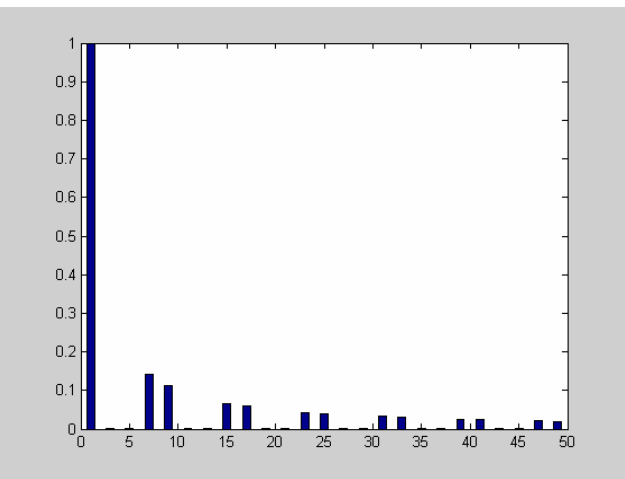

Fig. 7. Spectrum of $f_{\Psi 1}(x)$ : THD $=23,06 \% ; \delta=0,0252$ 


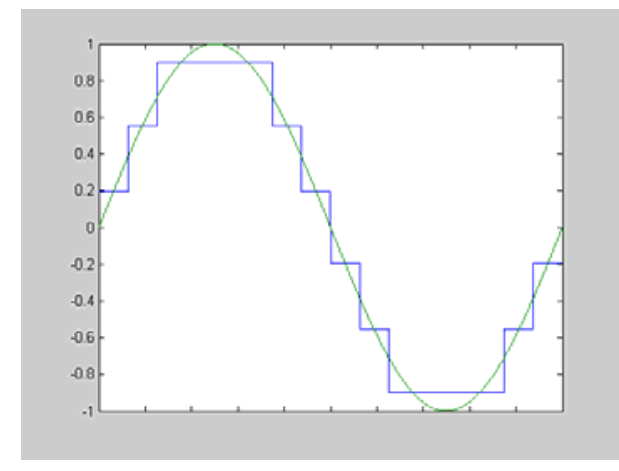

Fig. 8. Waveform of function $f_{\Psi_{2}}(x)$.

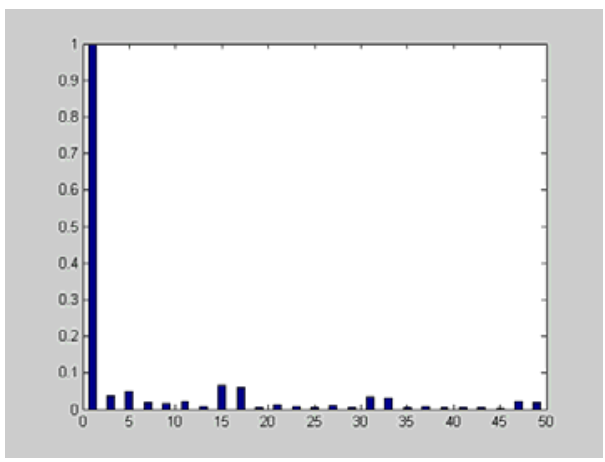

Fig. 9. Spectrum of $f_{\Psi_{2}}(x)$ : THD $=13,70 \% ; \delta=0,0091$.

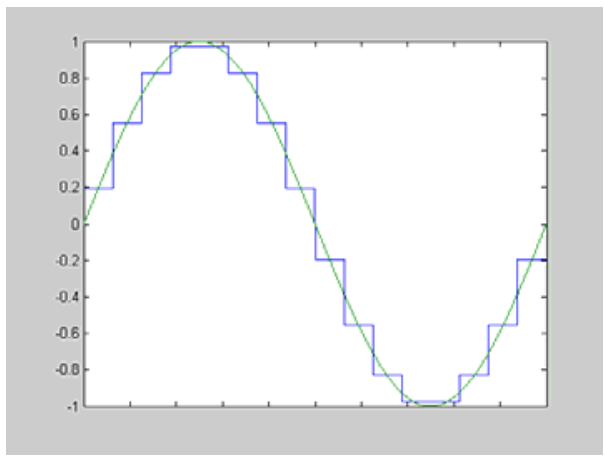

Fig. 10. Waveform of function $f_{\Psi_{3}}(x)$.

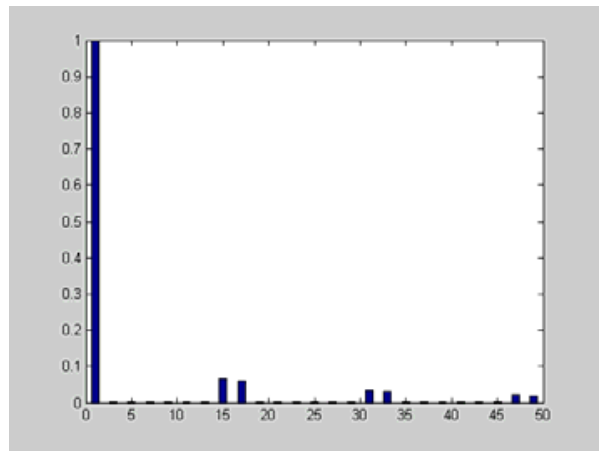

Fig. 11. Spectrum of $f_{\Psi 3}(x): T H D=11,44 \% ; \delta=0,0065$.

Selected parameters describing features of the wavelet applications in shaping stepped waveforms have been collected in Table 2. Besides average square errors $\delta$ and THD factors a $N_{\left|f_{\psi_{k}}\right|}$ parameter has been included. It denotes a number of different absolute values in one period of the stepped waveform. This parameter relates directly to the number of demanded DC voltage or cur- rent sources. The angle $\alpha$ denotes the length of a shortest step of the waveform and is equal to a half scale of the shortest wavelet applied for approximation. Function $f_{\psi 0}$ signifies one period of a rectangular wave which can be treated as the lowest step of approximation.

TABLE 2: Fundamental parameters of approximating functions $f_{\Psi 0}, f_{\Psi 1}, f_{\Psi 2}, f_{\Psi 3}, f_{\Psi 4}$.

\begin{tabular}{||c||c|c|c|cc||}
\hline \hline$f_{\Psi k}$ & $\alpha$ & $N_{\mid f_{\Psi_{k}}}$ & $\delta_{\psi k}$ & THD \\
\hline \hline$f_{\Psi 0}$ & $\pi$ & 1 & 0,0947 & 48,37 & $\%$ \\
\hline$f_{\Psi 1}$ & $\pi / 4$ & 2 & 0,0252 & 23,06 & $\%$ \\
\hline$f_{\Psi 2}$ & $\pi / 8$ & 3 & 0,0091 & 13,70 & $\%$ \\
\hline$f_{\Psi 3}$ & $\pi / 8$ & 4 & 0,0065 & 11,44 & $\%$ \\
\hline$f_{\Psi 4}$ & $\pi / 16$ & 8 & 0,0016 & $5,73 \%$ \\
\hline
\end{tabular}

\section{Comparative analysis}

Data gathered in Table 2 summarize the approach to wavelet synthesis - the expansion of the function $f(x)=\sin (x)$ by use of wavelet series. It permits to compare the features of the wavelet expansion with a typically used form of stepped waveforms in multilevel inverters. A cascade multilevel inverter where cells are equal each other, will determine a reference object [8]. The DC link voltage is divided by number of cells in one phase leg of the inverter and each cell is active during the same interval of time. So it is assumed here that waveforms generated by such an inverter are composed of steps of the same voltage and the same length $\alpha$. The angle length $\alpha$ of steps is determined by a number $N$ of steps in one period of the output waveform denoted as $F_{N}$. A comparison of parameters permitting to confront classic and wavelet waveforms is presented in Table 3.

Data concerning the standard model are denoted by index $N$ while data of the wavelet model - by index $\psi$. Remaining symbols have the following meaning:

- $\alpha_{\text {min }}-$ the shortest step angle,

- $N_{D C}$ - number of demanded DC sources,

- $\delta$ - approximation error,

- THD - total harmonic distortion factor.

TABLE 3: Comparative parameters of two models of AC waveform approximation.

\begin{tabular}{|c|c|c|c|c|c|c|c|c|c|}
\hline \multicolumn{2}{|c|}{$f_{\psi k} \cup F_{N}$} & \multicolumn{2}{|c|}{$\alpha_{\min }$} & \multicolumn{2}{|c|}{$N_{D C}$} & \multicolumn{2}{|c|}{$\delta$} & \multicolumn{2}{|c|}{$T H D$} \\
\hline$f_{\Psi k}$ & $F_{N}$ & $f_{\varphi k}$ & $F_{N}$ & $N_{\left|f_{\psi_{k}}\right|}$ & $N_{\left|F_{N}\right|}$ & $\delta_{\psi k}$ & $\delta_{N}$ & $f_{Y \psi_{k}}$ & $F_{N}$ \\
\hline$f_{\Psi_{0}}$ & $F_{N=2}$ & $\pi$ & $\pi$ & 1 & 1 & 0,0947 & 0,0947 & $48,37 \%$ & $48,37 \%$ \\
\hline$f_{\psi 1}$ & $F_{N=6}$ & $\pi / 4$ & $\pi / 3$ & 2 & 2 & 0,0252 & 0,0440 & $23,60 \%$ & $31,09 \%$ \\
\hline$f_{\psi_{2}}$ & $F_{N=12}$ & $\pi / 8$ & $\pi / 6$ & 3 & 3 & 0,0091 & 0,0113 & $13,70 \%$ & $15,23 \%$ \\
\hline$f_{\Psi 3}$ & $F_{N=16}$ & $\pi / 8$ & $\pi / 8$ & 4 & 4 & 0,0065 & 0,0064 & $11,44 \%$ & $11,41 \%$ \\
\hline$f_{\Psi 4}$ & $F_{N=24}$ & $\pi / 16$ & $\pi / 12$ & 8 & 6 & 0,0016 & 0,0028 & $5,73 \%$ & $7,63 \%$ \\
\hline
\end{tabular}

The comparison of parameters confirms that for the same number of independent sources the THD factor 
varies depending on the used model. Wavelet synthesis has advantages in relation to the standard solution when considering on lower levels of approximation.

On the second step of expansion the wavelet model produces shorter angles $\alpha_{\min }: \alpha=\pi / 4$ related to $\pi / 3$ in the waveform $F_{N=6}$. and generates waveforms with significantly reduced $\delta$ and THD. In both models the converter needs two DC sources but in wavelet converters the Dc voltages are different. Thanks to dyadic scaling in wavelet transform definition (8) it results in elimination of third and fifth harmonic in $f_{\psi 1}$ spectrum. The THD value $23,06 \%$ reaches almost the lowest level available in three level inverters. In Fig. 12 and 13 two spectra of considered waveforms are presented: spectrum of the waveform generated in a standard three level inverter and spectrum of the waveform obtained in the wavelet converter.

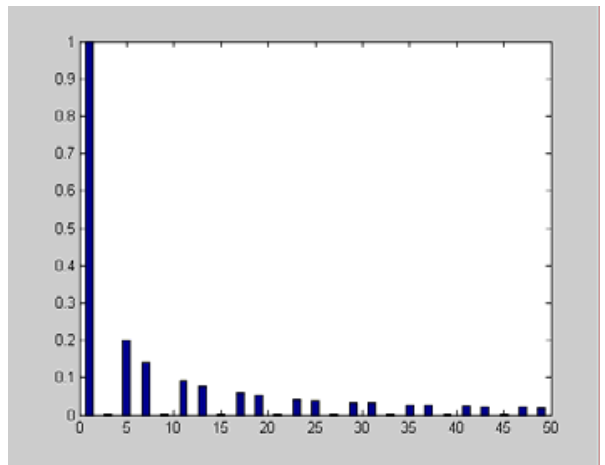

Fig. 12. Spectrum of $f_{N}(x)$ for $N=6$ : $T H D=31,09 \% ; \delta=0,0440$.

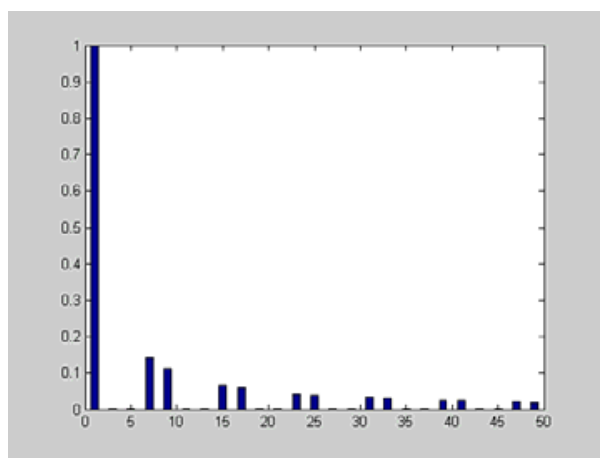

Fig. 13. Spectrum of: $f_{\psi 1}(x): \mathrm{THD}=23,06 \% ; \delta=0,0252$.

The next step of approximation permits to obtain also better waveforms in wavelet converter than in standard one. Taking as a criterion the aforementioned parameters $\alpha_{\min }, \delta$ and THD factor it is possible to compare adequately: $\pi / 8$ versus $\pi / 6,0,0091$ to 0,0113 and $13,70 \%$ to $15,23 \%$.

Following steps of wavelet approximation bring similar results as received in case of considered standard stepped waveforms. However it seems that the wavelet converter presents better possibilities with regard to control of fundamental voltage and frequency of output waveforms because it is equipped in diversified DC sources and can operate with shorter pulses. Although the problem is very important in AC drives control but is not considered in this paper.

\section{Wavelet converter}

One-phase wavelet voltage converter, generating $f_{\psi 1}$ waveform, consists of two voltage inverters (respectively transistors $T 1 \div T 4$ and $T 5 \div T 8$ ) connected in cascade. In each phase of the complex converter the component inverters are supplied from two independent voltage sources: $U_{\mathrm{D} 1}$ and $U_{\mathrm{D} 2}$. The supply voltages are proportional to the relevant amplitudes of component wavelets. For the converter output voltage of $500 \mathrm{~V}$ the voltage $U_{\mathrm{D} 1}=318 \mathrm{~V}$, and $U_{\mathrm{D} 2}=132 \mathrm{~V}$. The schematic diagram of three-level three-phase converter, consisting of three onephase component inverters is presented in Fig. 14.

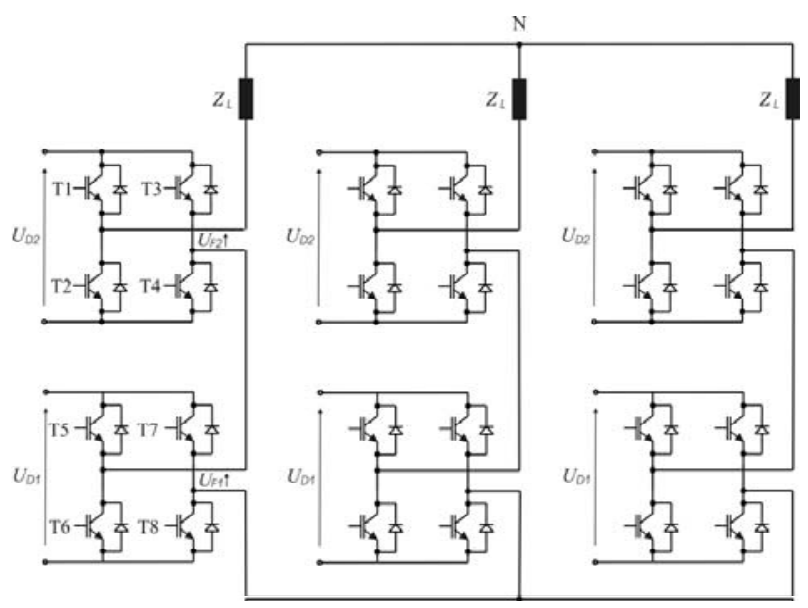

Fig. 14. Three-phase three-level converter as an example of wavelet theory application.

The control of such a converter is derived from described wavelets model. The inverter consisting of transistors $T 5 \div T 8$ generates waveform $U_{F l}$, corresponding with wavelet $f_{00}$, in each period of output voltage. It means that transistors $T 5$ and $T 8$ are switched on in the first half of the period while transistors $T 6$ and $T 7$ are switched on in the second one. The inverter consisting of transistors $T 1 \div T 4$ generates four waveform $U_{F 2}$, corresponding with wavelets $f_{-2 n}$, in each period of output voltage. Because the wavelets in the middle of the output waveform have a different phase shift than the other ones, the periodical switching pattern is as following: $T 2$ T3, T1-T4; T1-T4, T2-T3; T1-T4, T2-T3; T2-T3, T1-T4.

The summing process of the wavelets in cascade is accomplished by serial connection of the outputs of component inverters. When the transformer is used as a summing node the frequency corresponding with wavelets $f_{-2 n}$ is $200 \mathrm{~Hz}$. Anyhow due to the phase change there will appear pulses of $5 \mathrm{~ms}$ duration two times in each output voltage period.

It is worth mentioning, that the power rating of the component inverters is not equal. The power of the inverter generating the wavelets $f_{-2 n}$ (transistors $T 1 \div T 4$ ) is much lower than the power of the second one generating wavelet $f_{00}$ (transistors $T 5 \div T 8$ ).

The topology of multilevel converter is dependent on the method used for its output waveform synthesis. The proposed method based on wavelet transform is very suitable for cascade converters even so it demands independent voltages sources. 


\section{Conclusions}

Advantageous features of wavelets permit to use them not only for analysis of processes consisting in decomposition but on the contrary in composition of signals. The wavelets may be also applied in composition of signals and structures in power electronics. The paper presents a theory of waveform synthesis based on wavelets transform which may be a useful mathematical tool in designing of multilevel converter structures and control strategies.

The wavelet transform synthesis applied to control multilevel converters has good features in relation to standard methods. The wavelet converter becomes automatically an asymmetric multilevel inverter because it assures better and faster adaptation of output waveforms to sine wave shape. For low level of approximation including three and four level inverters, the wavelet synthesis proves this feature and permits to obtain better approximation of waveforms. Furthermore the wavelet control method has the following features:

- low harmonic content of output waveforms,

- relatively low switching frequency of all inverters,

- diversified power rating of component inverters,

- simple converter control circuit.

The mathematical wavelet model of converter is based on discrete inverse wavelet transform. Thanks to its universality the model gives an impact for development of converter topologies and control strategies. It may be developed by incorporating suitable control functions e.g. PWM thus permitting to be applied in $\mathrm{AC}$ drives.

The possible application area belongs mainly to higher power converters especially in high power uninterruptible power supplies, voltage regulators like FACTS and UPQC, distributed power generation systems with DC sources like photovoltaic or fuel cells with discrete voltage levels. In the sources of this kind there is an easy way to group cells to obtain the desired voltage levels for the multilevel converter.

\section{Acknowledgement}

The scientific work was supported by The Polish Ministry of Science and Higher Education in years 2006 - 2009 as a research project.

\section{References}

[1] Akagi H.: Control Strategy and Sections of a Shunt Active Filter for Damping of Harmonic Propagation in Power Distribution Systems. IEEE TRAN. on Power Delivery, January 1997.

[2] Blaabjerg F., Jaeger U., Munk-Nielsen S., Pedersen J. K.: Power Losses in PWM_VSI Inverter Using NPT or PT IGBT Devices. IEEE Transactions on Power Electronics, vol. 10, no. 3, May 1995.

[3] Białasiewicz J. T.: Falki i aproksymacje. Wydawnictwa Naukowo-Techniczne, Wydanie drugie, Warszawa 2004.
[4] Chiasson J. N., Tolbert L. M., McKenzie K. J., Zhong Du.: Control of a Multilevel Using Resultant Theory. IEEE Transactions on Control Systems Technology, vol. 11, no. 3, May 2003.

[5] Daubechies I.: The wavelet Transform, time-frequency localization and signal analysis. IEEE Transactions on Informatics Theory, 1990, vol. 36, s. 961-1005.

[6] Faranda R., Valade I.: UPQC Compensation Strategy and Design Aimed at Reducing Losses. IEEE International Symposium on Industrial Electronics ISIE 2002, vol. 4.

[7] Graps A.: An Introduction to Wavelets, IEEE Computational Science and Engineering, summer 1995, vol. 2, no. 2.

[8] Haar A.: Zur Theorie der orthogonalen Funktionensysteme, Mathematische Annalen, 1910, Vol. 69, pp. 331371.

[9] Hashad M., Iwaszkiewicz J.: A Novel OrthogonalVectors-Based Topology of Multilevel Inverters, IEEE Transactions on Industrial Applications, vol. 49, no. 4, August 2002.

[10] Iwaszkiewicz J., Hashad M.: Convertor Control with Help of Orthogonal Space Vectors - Novel Control Strategy and Novel Topology, PCC Osaka 2002.

[11] Iwaszkiewicz J.: A Novel Recurrent Approach to the Output Voltage Filtering of the Inverter, EPQU 2003.

[12] Iwaszkiewicz J., Perz J.: Amplitude Modulation - an Alternative Method of Generating the Convertor Output Waveforms, ICREPQ'04, Barcelona 2004.

[13] Iwaszkiewicz J., Perz J. - „Multilevel Convertors for Distributed Power Generation Systems with DC Voltage Sources", International Conference on Renewable Energy and Power Quality ICREPQ'05, Saragossa 2005.

[14] Iwaszkiewicz J., Perz J. - „Mathematical Approach to THD Optimisation in Multilevel Convertors", International Conference on Renewable Energy and Power Quality ICREPQ'06, Palma de Mallorca 2006.

[15] Iwaszkiewicz J., Perz J. - „A Novel Approach to Sinewave Approximation in Multilevel Converters", The IEEE Industry Applications Society Workshop on Renewable Energy Based Units and Systems (REBUS), St. Petersburg 2006.

[16] Iwaya K., Takahashi I.: Novel Multilevel PWM Wave Control Method using Series Connected Full Bridge Inverters, Proceedings of the IEEE Electric Machines and Drives Conference, volume 3, pages 1543-1548, June 2003.

[17] Kawabata T., Kawabata Y., Nishiyama K.: New Configuration of High-Power Inverter Drives," Proceedings of the IEEE International Symposium on Industrial Electronics, volume 2, pages $850-855$, June 1996.

[18] Kaźmierkowski M., P., F. Blaabjerg F.: Impact of Emerging Technologies on PWM Control of Power Electronic s, IEEE Industrial Electronics Society Newsletter, December, 1995.

[19] Lida S., Masukawa S., and Kubota Y.: Improved Voltage Source Inverter With 18-Step Output Waveforms, IEEE Industry Applications Magazine, January/February 1998.

[20] Rademacher H.: Einige Satze von allgemeinen orthogonalfunktionen. Mathematische Annalen, 1922, pp. 122 138.

[21] Rodriguez J., Lai J.-S., Peng F.Z..: Multilevel Inverters: A Survey of Topologies, Controls and Applications, IEEE Transactions on Industrial Electronics, vol. 49, no. 4, pp. 724-738, August 2002.

[22] Sten K., Stacey E.: UPFC-Unified Power Flow Controller: Theory, Modeling, and Applications, IEEE Transactions on Power Delivery. Vol. 13. No. 4. October 1998.

[23] Walsh J. L.: A closed set of orthonormal functions. Amer. Journal of Mathematics, 1923, no. 1 pp. 5-24. 